\title{
Awareness of Diabetic Patients Regarding the Bidirectional Association between Periodontal Disease and Diabetes Mellitus: A Public Oral Health Concern
}

\author{
Allauddin Siddiqi ${ }^{1}$, Sobia Zafar ${ }^{2}$, Ajay Sharma $^{3}$, Alessandro Quaranta ${ }^{4}$
}

\begin{abstract}
Aim and objective: The literature regarding the perception of the two-way relationship between diabetes mellitus and periodontal disease representing diabetic patients living in Australia is scarce. The study aimed to evaluate the knowledge and attitudes of diabetic patients regarding the bidirectional link and the role of medical healthcare professionals in providing oral health advice to their patients.

Materials and methods: A convenience sample of diabetic patients attending general practice-based medical and dental centers was invited to complete a questionnaire-based survey. The survey was anonymous, and the responses of the participants were not identifiable.

Results: A total of 241 participants completed the questionnaire; however, three survey responses were excluded as most of the critical questions were not answered. The majority (87.81\%) of the participants reported with type 2 diabetes mellitus, while $11.76 \%$ had type 1 diabetes mellitus. Just over $61 \%$ of the participants reported brushing their teeth twice a day. The majority of participants (66.38\%) said that their medical practitioner/diabetic educator never asked or examined their oral hygiene or any issues with the gums or teeth. The study noted that $54 \%$ of the participants never received any information regarding the bidirectional relationship between periodontal disease and diabetes mellitus and were unaware of the association.

Conclusion: Patients with diabetes mellitus lack knowledge of the bidirectional association between periodontal disease and diabetes mellitus. In this regard, the study urged the need to implement European Federation of Periodontology and International Diabetes Federation guidelines effectively. Medical healthcare professionals and dentists should provide mutual care and should consider every patient as a shared responsibility. Clinical significance: Early detection of the disease, timely referrals, and a collaborative approach will enhance patient care and improve the quality of life of individuals living with periodontal disease.
\end{abstract}

Keywords: Bidirectional association, Collaborative care, Diabetes mellitus, Periodontal disease.

The Journal of Contemporary Dental Practice (2020): 10.5005/jp-journals-10024-2974

\section{INTRODUCTION}

Diabetes mellitus and periodontal disease are chronic inflammatory conditions, destructive and progressive in nature. They are considered as global health concerns due to their increasing prevalence. ${ }^{1}$ Approximately 1 in 11 adults aged 20-79 years are living with diabetes mellitus. ${ }^{2}$ Similarly, according to recent reports, the global prevalence of periodontitis is $11.2 \% .^{3}$ Diabetes and periodontal diseases are considered as lifestyle disorders, and the basis of the management of these conditions is self-management. ${ }^{4}$

Strong evidence endorses the bidirectional relationship between periodontal disease and diabetes mellitus. ${ }^{5,6}$ Through some positive lifestyle modifications, these conditions can be prevented. However, an essential aspect of self-management and prevention from progressive destruction is the motivation and awareness of the associated risk factors by the patients. ${ }^{7} \mathrm{~A}$ recent cross-sectional study from Spain found that approximately $38 \%$ of the participants were unaware of the term "periodontitis/ pyorrhoea". ${ }^{8}$ Sandberg and colleagues noted $85 \%$ of the participants in their study never received any information regarding the association between gum (periodontal) disease and diabetes mellitus. ${ }^{9}$

Health literacy, including oral health awareness, plays a crucial role in practicing preventive measures, as does seeking early help for the management of the disease. ${ }^{10}$ Schillinger et al., ${ }^{11}$ in a crosssectional analysis of 408 patients with type 2 diabetes mellitus (T2DM), investigated the relationship between health literacy and
${ }^{1}$ Department of Periodontics and Implantology, School of Dentistry and Oral Health, Griffith University, Griffith Health Centre (G40), Gold Coast, Australia

${ }^{2}$ Department of Paediatric Dentistry, School of Dentistry, The University of Queensland, Herston, Australia

${ }^{3}$ School of Dentistry and Oral Health, Griffith University, Griffith Health Centre (G40), Gold Coast, Australia

${ }^{4}$ Department of Periodontics, Sydney Dental Hospital, NSW, Australia

Corresponding Author: Allauddin Siddiqi, Department of Periodontics and Implantology, School of Dentistry and Oral Health, Griffith University, Griffith Health Centre (G40), Gold Coast, Australia, Phone: +61404111998, e-mail: siddiqidr@gmail.com

How to cite this article: Siddiqi A, Zafar S, Sharma A, et al. Awareness of Diabetic Patients Regarding the Bidirectional Association between Periodontal Disease and Diabetes Mellitus: A Public Oral Health Concern. J Contemp Dent Pract 2020;21(11):1270-1274.

Source of support: Oral hygiene products (tooth paste, tooth brush and mouth rinse) for the participants were kindly provided by ColgatePalmolive, Australia

Conflict of interest: None

diabetes' outcomes. They found that inadequate health knowledge was related to poorer glycemic control that further leads to a substantial burden of diabetes-related complications. ${ }^{11}$ 
Reports suggest that patients with diabetes mellitus and periodontal disease hold a limited understanding of the etiology of the disease, the factors that could increase the risk of complications, and the potential appropriate measures in the prevention of the related conditions. ${ }^{12}$ Wide gaps in oral health literacy exist mainly regarding the causal and allied factors of periodontal disease, and the link with systemic disorders, particularly diabetes. There is a need to investigate, at the local/community level, the prevalence of and the barriers to the integration of oral-general health literacy for the general public, especially patients with diabetes mellitus. To the authors' knowledge, there is no literature available on the education and awareness of the bidirectional link that represents diabetic patients living in Australia. Therefore, the present crosssectional study aimed to evaluate the knowledge and attitude of diabetic patients about the two-way relationship and the role of medical healthcare professionals in providing oral health advice to their patients to identify gaps in the dissemination of this critical information.

\section{Materials and Methods}

Ethical approval was obtained from the Human Research Ethics Committee of the institution, and participants gave consent to be included in the study. A convenience sample of diabetic patients (type 1 and type 2) attending general practice-based medical (diabetic educators/nurses) and dental centers (patients attending dental schools) was invited to complete the questionnaire. The questionnaire was designed to investigate the knowledge and understanding of periodontal disease and the link between periodontal disease and diabetes mellitus among patients with diabetes mellitus. The survey was created by a panel of experts who have experience in research methods to assess the relevancy, clarity, simplicity, and necessity of the questions (Table 1). The survey questions were finalized after initial testing (piloting) on a group of diabetic patients and conducting a systematic review of the literature that investigated the medical healthcare professionals' knowledge and understanding of the bidirectional link. ${ }^{12}$ The systematic review also identified that the data from Australia are missing. The questionnaire was compiled in the lay language without any confusion or ambiguity, thus avoiding any potential response bias. Participants were also offered help in completing the survey when required. Most items in the survey were in multiple-choice format, with options of "other" where the participants could elaborate further in text free fields if they deemed it appropriate.

\section{Study Sample}

Only patients with a confirmed diagnosis of diabetes mellitus (6 months before completing the questionnaire) were included in the study. Patients who were recently diagnosed with diabetes $(<6$ months) were not included in the study. The survey was anonymous, and the responses of the participants were not identifiable.

\section{Data Analysis}

The responses were collated electronically on Qualtrics and transferred onto an Excel ${ }^{\circledR}$ spread-sheet (Microsoft Corp., Redmond, WA, USA) and analyzed using a commercially available statistical software package (IBM SPSS ${ }^{\circledR}$ Statistics for Windows, Version 27.0. Armonk, NY, USA: IBM Corp). The bivariate analyzes using Fisher's exact and chi-square test (in SPSS ${ }^{\circledR} 27$ ) were utilized to analyze whether participants' awareness regarding the bidirectional
Table 1: Summary of the survey questionnaire

\begin{tabular}{ll}
\hline Category & Number of questions and the related theme \\
\hline 1 & $\begin{array}{l}\text { It consisted of } 12 \text { items that recorded biographic and } \\
\text { demographic data (age, gender, marital status, location, } \\
\text { education, employment). }\end{array}$ \\
& $\begin{array}{l}\text { Consisted of five questions regarding smoking history } \\
\text { and any allergies. } \\
\text { Consisted of } 10 \text { items that record information regarding } \\
\text { diabetes mellitus including; type of diabetes, age at } \\
\text { diagnosis, family history, } \mathrm{A}_{1 \mathrm{C}} \text { status, type of medications } \\
\text { in use for diabetes. } \\
\text { Consisted of } 13 \text { items that record participant's oral } \\
\text { hygiene habits (brushing frequency, use of floss and } \\
\text { interdental devices, the mouth used). Visit the dentist } \\
\text { and the reason for the visit, bleeding gums, bad breath, } \\
\text { sensitive teeth, pain, family history of gum disease, and } \\
\text { self-precede oral hygiene). } \\
\text { Consisted of } 7 \text { items that referred to the patient's } \\
\text { medical professional's attitude toward oral health } \\
\text { information (regarding the bidirectional relationship } \\
\text { between diabetes and periodontal disease. }\end{array}$ \\
5
\end{tabular}

association was associated with age, gender, educational level, and medical professional's clinical practice. A $p$ value of $<0.05$ was considered to be statistically significant. The output of data was presented in a table format (total responses and percentage) as well as in a graphical format.

\section{Results \\ Demographic Data and Smoking History}

A total of 241 participants completed the questionnaire; however, three survey responses were excluded as most of the critical questions were not answered. Hence, 238 participants were included in the data analysis. Male to female ratio was not statistically significant, with $46.86 \%$ female and $53.14 \%$ male participants with the age range of 30-77 years. Sixteen participants were of Aboriginal and/or Torres Strait Islander origin. The majority of participants (44.3\%) had primary to undergraduate qualifications, while approximately $20 \%$ of participants held a postgraduate qualification. Employment status revealed that $43 \%$ of the participants were retired and approximately $11 \%$ were unemployed. About $17 \%$ of the participants were smokers, and $20 \%$ were ex-smokers. Among smokers, 58\% were willing to quit smoking.

The majority (87.81\%) of the participants reported T2DM, while $11.76 \%$ had T1DM. Approximately $58 \%$ of the participants reported a family history of diabetes either from the mother's or the father's side, while approximately $10 \%$ were unsure of their family history of diabetes. Regarding the management/control of diabetes (wellcontrolled-yes/no/uncertain), 15\% reported not well-controlled diabetes. When asked about the medication they used to manage diabetes [insulin, tablets (antihyperglycemic), tablets and insulin (both)], and control with diet only to manage diabetes, $68.2 \%$ used oral anti-hyperglycemic medication to control diabetes. On the contrary, $16 \%$ of the participants used both insulin and oral antihyperglycemic medication for the management of their diabetes. Almost $6 \%$ of participants managed their diabetes only with diet control. Just over $64 \%$ of participants also reported having other medical conditions, including hypertension, osteoporosis, cancer, kidney disorders, and depression. 


\section{Participants' Oral Hygiene Habits}

Around $81 \%$ of participants reported attending the dentist during the past 12 months. Several reasons for visiting the dentist were mentioned, including extractions due to infection, gum treatment, restorative work, dentures, and pain. On the one hand, $43 \%$ of the patients were having supportive periodontal therapy. On the other hand, $31 \%$ of participants had not received any periodontal assessment and/or management over the last 2 years. Just over $16 \%$ of participants also reported having a family history of periodontitis, while $27.6 \%$ were unsure of this relationship.

Concerning toothbrushing frequency, $61.4 \%$ of the participants reported brushing their teeth twice a day. Just over $28 \%$ of the participants never used floss for cleaning interdental spaces. Similarly, almost half of the participants never used interdental brushes for cleaning, and around 38\% of participants never used mouth rinse as an oral hygiene measure.

Bleeding gums were reported by $1 / 3$ of the participants, and a similar frequency of participants also reported halitosis (selfperceived bad breath). Pain associated with gums was reported by $23 \%$ of the participants, and almost $42 \%$ said they have hypersensitive teeth. Concerning the self-perceived oral hygiene status, $46 \%$ of the participants rated their oral hygiene as poor to fair, while only $7.7 \%$ thought they have excellent oral health.

Diabetic patients and medical healthcare professionals' attitudes toward oral health information (regarding the bidirectional relationship between diabetes and periodontal disease)

The majority of participants $(66.38 \%)$ reported that their medical practitioner/diabetic educator never asked about or examined their oral hygiene or any issues with the gums or teeth (Table 2 ). Only $11.21 \%$ of medical practitioners/diabetic educators inquired about the oral health of their patients. Similarly, almost half of the participants recorded that their medical practitioner/diabetic educator never advised them to take good care of their oral hygiene. On the contrary, only $13.37 \%$ always provided oral health advice.

The study noted that $54 \%$ of the participants never received any information regarding the bidirectional relationship between periodontal disease and diabetes mellitus and were unaware of the association (Table 2 ). While only $36.5 \%$ of participants reported receiving this information, in this regard, only $10.85 \%$ of medical professionals conveyed this information to the participants. In comparison, $41 \%$ of the dentists and $18.86 \%$ of diabetic educators were the sources of this information. Electronic media and newspapers accounted for $3.42 \%$ in providing this information.

\section{Discussion}

The results of this cross-sectional investigation demonstrated that diabetic patients at large are not receiving oral health advice, particularly relating to periodontal disease and diabetes mellitus relationship. Hence, around half of the participants were unaware of this association. Although the participants regularly consulted their medical healthcare professionals for the management of their diabetes and general health, only $10.85 \%$ of participants received this information from their medical practitioners. Furthermore, only $11.21 \%$ of medical practitioners/diabetic educators examined or discussed oral health with their patients, meaning a large number of medical healthcare professionals are not giving importance to this aspect of health.

The results of the present study corroborate those of other reports by Orlando et al., ${ }^{13}$ Bahammam, ${ }^{14} \mathrm{Al}$ Habashneh et al., ${ }^{15}$
Table 2: Medical healthcare professional's attitude toward oral health information (regarding the bidirectional relationship between periodontal disease and diabetes mellitus)

\begin{tabular}{|c|c|c|}
\hline & $\%$ & \# \\
\hline \multicolumn{3}{|c|}{ Are you under the care of a general medical practitioner? } \\
\hline - Yes & 97.01 & 227 \\
\hline \multirow[t]{3}{*}{ - No } & 2.99 & 7 \\
\hline & Answered & 234 \\
\hline & Skipped & 7 \\
\hline \multicolumn{3}{|c|}{$\begin{array}{l}\text { Did your medical practitioner/diabetic specialist ever ask about } \\
\text { your gums/oral health or checked your gums/teeth? }\end{array}$} \\
\hline - No & 66.38 & 154 \\
\hline - Occasionally & 22.41 & 52 \\
\hline \multirow[t]{3}{*}{ - Always } & 11.21 & 26 \\
\hline & Answered & 232 \\
\hline & Skipped & 9 \\
\hline \multicolumn{3}{|c|}{$\begin{array}{l}\text { Did your medical practitioner/diabetic specialist ever instruct you to } \\
\text { take good care of your gums and teeth? }\end{array}$} \\
\hline - Never & 48.28 & 112 \\
\hline - Occasionally & 19.83 & 46 \\
\hline - Sometimes & 7.76 & 18 \\
\hline - Most of the times & 10.34 & 24 \\
\hline \multirow[t]{3}{*}{ - Always } & 13.79 & 32 \\
\hline & Answered & 232 \\
\hline & Skipped & 9 \\
\hline \multicolumn{3}{|c|}{$\begin{array}{l}\text { Have you ever been instructed in oral hygiene measures by a den- } \\
\text { tist/hygienist/medical practitioner/diabetic educator? }\end{array}$} \\
\hline - Yes & 54.11 & 125 \\
\hline - No & 33.33 & 77 \\
\hline \multirow[t]{3}{*}{ - Do not remember } & 12.55 & 29 \\
\hline & Answered & 231 \\
\hline & Skipped & 10 \\
\hline
\end{tabular}

Weinspach et al., ${ }^{16}$ and Allen et al. ${ }^{17}$ where $44-55 \%$ of diabetic patients were aware of the perio-diabetes association. These results are slightly higher when compared with a recent systematic review that indicated that $27 \%$ of diabetic patients were aware of the bidirectional association. ${ }^{12}$

Another key finding of the present study was the lack of communication and discussion about oral health and its implications for systemic health between the medical healthcare professional and the diabetic patient. A low number of diabetic patients $(10.85 \%)$ reported that their medical healthcare professional discussed oral health, especially the relationship between diabetes and periodontal disease. This was also reflected in clinical practice, as only $15 \%$ of medical professionals referred their diabetic patients for a dental consultation as reported in a recent study. ${ }^{12}$ On the contrary, only $41 \%$ of the dentists discussed the periodontal-diabetes disease link with their patients. These are alarming results as dentists are considered as the vital source of information regarding periodontal/oral health and diabetes mellitus. The situation demonstrates that dentists are not effectively playing their part in the oral health education of diabetic patients.

Several cross-sectional and longitudinal studies have established that oral health is significantly affected in patients with uncontrolled diabetes mellitus, with resultant attachment loss and 
ultimately, tooth loss. ${ }^{18-20}$ Hence, oral hygiene practice has been stressed to avoid significant complications associated with the two disorders. The present study noted a higher proportion (69\%) of participants brushed their teeth twice a day, whereas almost half $(49.55 \%)$ of the participants never used interdental brushes for cleaning interdental spaces. A similar trend has been reported in other studies by Al-Amassi and Al-Dakheel. ${ }^{21}$ They noted only $10.4 \%$ of the participants in their research used interdental brushes as an oral hygiene aid, representing ineffective oral hygiene practices. ${ }^{21}$

Diabetic patients are more prone to have periodontal disease with an odds ratio of 2-3 compared with a healthy person. ${ }^{22-24}$ This high rate of occurrence of periodontal disease has been attributed to the increased tendency of infection and bacterial growth in deep pockets and impaired healing. ${ }^{25,26} \mathrm{~A}$ recent study reported higher values of $\mathrm{HbA} 1 \mathrm{c}$ in patients with periodontal disease (periodontitis) compared with patients without periodontal disease. ${ }^{27}$ Similarly, $18 \%$ of patients with severe periodontal disease were reported as prediabetic (un-diagnosed cases of diabetes mellitus), confirming that severe periodontitis could be an early sign of diabetes mellitus. ${ }^{27}$ The authors suggested that screening for diabetes, especially at dental clinics that manage periodontal patients, would be a suitable and cost-effective way. ${ }^{27}$

The data did not identify any correlation concerning the self-reported $\mathrm{HbA1c}$ levels and the awareness of the bidirectional association between periodontal disease and diabetes mellitus. This is mainly because participants variably reported their $\mathrm{HbA} 1 \mathrm{C}$ levels. In the present investigation, $33.77 \%$ of participants reported bleeding gums while brushing, and a similar rate of participants also reported having halitosis. However, it can be hypothesized that in those participants, the periodontal disease would be in process, and their $\mathrm{HbA1c}$ values would have been on the higher side, as $15 \%$ of the participants reported not having their diabetes well-controlled. Furthermore, approximately $13.36 \%$ of the participants reported having poor oral hygiene.

Keeping in mind the high risk of periodontal disease among diabetic patients, and its influence on the stability of glycemic levels, the knowledge of the bidirectional link is low. This lack of awareness is reflected in the oral health behavior and attitudes of diabetic patients. In this regard, medical healthcare professionals and dentists are not effectively playing their role to disseminate the information to their patients. The study had its limitations because of its small sample size and the potential for biased reporting by the participants. Nevertheless, the study provided critical data representing Australia that showed the knowledge and attitudes of diabetic patients.

The recent consensus report and guidelines by European Federation of Periodontology (EFP) and International Diabetes Federation (IDF) suggest that oral health education should be part of the overall education program of all diabetic patients, and medical healthcare professionals (physicians) should ask about their periodontal status. ${ }^{6}$ In this regard, an initial history of bleeding gums, loose teeth, flaring or spreading of teeth, halitosis, and gingival abscesses are positive indicators of periodontal disease that could direct physicians to refer their patients for a dental consultation. Physicians and dentists should be proactive in the initial diagnosis of periodontal disease and diabetes mellitus. The guidelines are there, and all we need to do is to accurately follow them for the optimized care of our diabetic patients.

Over the last few years, the EFP and IDF have taken serious incentives to improve the oral health literacy of individuals, and diabetic patients in particular. Quite recently, EFP in an outreach project launched the Perio and Diabetes campaign on World Diabetes Day to give scientific credibility to this critical issue and has taken a holistic approach toward oral health education. So far, there has been a significant development in improving awareness regarding the periodontal disease-diabetes association. Embracing such measures on a larger scale will help us to curb the global burden of periodontal disease and diabetes mellitus at the same time. Early detection of the disease, timely referrals, and a collaborative approach will enhance patient care and improve the quality of life of individuals living with periodontal disease. ${ }^{28}$

\section{Conclusion}

Within the limitations of the study, it can be concluded that patients with diabetes mellitus lack the knowledge of the bidirectional association between periodontal disease and diabetes mellitus. In this regard, the study urged the need to effectively implement EFP and IDF guidelines. Medical healthcare professionals and dentists should provide mutual care and should consider every patient as a shared responsibility. Now, more action is needed to disseminate this critical information to diabetic patients effectively.

\section{ACKnOWLedgments}

The authors would also like to thank the participants for their time. Special thanks to Dr Susan Cartwright (Scientific Affairs Manager, South Pacific, Colgate-Palmolive Company) for providing oral hygiene products for the participants, Dr Muhammad Yasir (IT Consultant for the construction of the online survey), and Mr Mustafa Siddiqi for research data handling.

\section{References}

1. Peres MA, Macpherson LMD, Weyant RJ, et al. Oral diseases: a global public health challenge. Lancet 2019;394(10194):249-260. DOI: 10.1016/S0140-6736(19)31146-8.

2. Saeedi P, Petersohn I, Salpea P, et al. Global and regional diabetes prevalence estimates for 2019 and projections for 2030 and 2045 results from the international diabetes federation diabetes Atlas. 9th ed. Diabetes Res Clin Pract 2019;157:107843. DOI: 10.1016/j. diabres.2019.107843.

3. Jin LJ, Lamster IB, Greenspan JS, et al. Global burden of oral diseases: emerging concepts, management and interplay with systemic health. Oral Dis 2016;22(7):609-619. DOI: 10.1111/odi.12428.

4. Syrjälä AM, Kneckt MC, Knuuttila ML. Dental self-efficacy as a determinant to oral health behaviour, oral hygiene and $\mathrm{HbA} 1 \mathrm{c}$ level among diabetic patients. J Clin Periodontol 1999;26(9):616-621. DOI: 10.1034/j.1600-051x.1999.260909.x.

5. Chapple IL, Genco R. Working group 2 of the joint EFP/AAP workshop. diabetes and periodontal diseases: consensus report of the joint EFP/ AAP workshop on periodontitis and systemic diseases. J Periodontol 2013;84(4 Suppl):S106-S112. DOI: 10.1902/jop.2013.1340011.

6. Sanz M, Ceriello A, Buysschaert M, et al. Scientific evidence on the links between periodontal diseases and diabetes: consensus report and guidelines of the joint workshop on periodontal diseases and diabetes by the International Diabetes Federation and the European Federation of Periodontology. J Clin Periodontol 2018;45(2):138-149. DOI: $10.1111 /$ jcpe.12808.

7. National Institute of Dental and Craniofacial Research, National Institute of Health, U.S. Public Health Service, Department of Health and Human Services. The invisible barrier: literacy and its relationship with oral health. A report of a workgroup sponsored by the National Institute of Dental and Craniofacial Research, National Institute of 
Health, U.S. Public Health Service, Department of Health and Human Services. J Public Health Dent 2005;65(3):174-182.

8. Varela-Centelles P, Diz-Iglesias P, Estany-Gestal A, et al. Periodontal awareness and what it actually means: a cross-sectional study. Oral Dis 2019;25(3):831-838. DOI: 10.1111/odi.13026.

9. Sandberg GE, Sundberg HE, Wikblad KF. A controlled study of oral self-care and self-perceived oral health in type 2 diabetic patients. Acta Odontol Scand 2001;59(1):28-33. DOI: 10.1080/ 000163501300035742.

10. Moore PA, Orchard T, Guggenheimer J, et al. Diabetes and oral health promotion: a survey of disease prevention behaviors. J Am Dent Assoc 2000;131(9):1333-1341. DOI: 10.14219/jada.archive.2000. 0388.

11. Schillinger $D$, Grumbach $K$, Piette J, et al. Association of health literacy with diabetes outcomes. JAMA 2002;288(4):475-482. DOI: 10.1001/ jama.288.4.475.

12. Siddiqi A, Zafar S, Sharma A, et al. Diabetic patients' knowledge of the bidirectional link: are dental health care professionals effectively conveying the message? Aust Dent J 2019;64(4):312-326. DOI: 10.1111/ adj.12721.

13. Orlando VA, Johnson LR, Wilson AR, et al. Oral health knowledge and behaviors among adolescents with type 1 diabetes. Int J Dent 2010;2010:942124. DOI: 10.1155/2010/942124.

14. Bahammam MA. Periodontal health and diabetes awareness among Saudi diabetes patients. Patient Prefer Adherence 2015;9:225-233. DOI: 10.2147/PPA.S79543.

15. Al Habashneh R, Khader Y, Hammad MM, et al. Knowledge and awareness about diabetes and periodontal health among Jordanians. J Diabetes Complications 2010;24(6):409-414. DOI: 10.1016/j. jdiacomp.2009.06.001.

16. Weinspach K, Staufenbiel I, Memenga-Nicksch S, et al. Level of information about the relationship between diabetes mellitus and periodontitis-results from a nationwide diabetes information program. Eur J Med Res 2013;18(1):6. DOI: 10.1186/2047783X-18-6.

17. Allen $\mathrm{EM}, \mathrm{Ziada} \mathrm{HM}, \mathrm{O}^{\prime}$ Halloran $\mathrm{D}$, et al. Attitudes, awareness and oral health-related quality of life in patients with diabetes. J Oral Rehabil 2008;35(3):218-223. DOI: 10.1111/j.1365-2842.2007.01760.x.
18. Emrich LJ, Shlossman M, Genco RJ. Periodontal disease in non-insulindependent diabetes mellitus. J Periodontol 1991;62(2):123-131. DOI: 10.1902/jop.1991.62.2.123.

19. Taylor GW, Burt BA, Becker MP, et al. Glycemic control and alveolar bone loss progression in type 2 diabetes. Ann Periodontol 1998;3(1):30-39. DOI: 10.1902/annals.1998.3.1.30.

20. Grossi SG, Genco RJ. Periodontal disease and diabetes mellitus: a two-way relationship. Ann Periodontol 1998;3(1):51-61. DOI: 10.1902/ annals.1998.3.1.51.

21. Al-Amassi BY, Al-Dakheel RS. Oral hygiene practice of adult diabetic patients and their awareness about oral health problems related to diabetes. J Dent Oral Hyg 2017;9(2):8-14. DOI: 10.5897/JDOH2017.0219.

22. Khader YS, Dauod AS, El-Qaderi SS, et al. Periodontal status of diabetics compared with nondiabetics: a meta-analysis. J Diabetes Complications 2006;20(1):59-68. DOI: 10.1016/j. jdiacomp.2005.05.006.

23. Khouja T, Miller RG, Moore PA, et al. Periodontal disease, smoking, cardiovascular complications and mortality in type 1 diabetes. J Diabetes Complications 2019;33(9):603-609. DOI: 10.1016/j. jdiacomp.2019.05.025.

24. Mealey $\mathrm{BL}$, Ocampo GL. Diabetes mellitus and periodontal disease. Periodontol 2000 2007;44(1):127-153. DOI: 10.1111/j.16000757.2006.00193.x.

25. Salvi GE, Carollo-Bittel B, Lang NP. Effects of diabetes mellitus on periodontal and peri-implant conditions: update on associations and risks. J Clin Periodontol 2008;35(8 Suppl):398-409. DOI: 10.1111/j.1600-051X.2008.01282.x.

26. Muller LMAJ, Gorter KJ, Hak E, et al. Increased risk of common infections in patients with type 1 and type 2 diabetes mellitus. Clin Infect Dis 2005;41(3):281-288. DOI: 10.1086/431587.

27. Teeuw WJ, Kosho MX, Poland DC, et al. Periodontitis as a possible early sign of diabetes mellitus. BMJ Open Diabetes Res Care 2017;5(1):e000326. DOI: 10.1136/bmjdrc-2016-000326.

28. Siddiqi A, Zafar S, Sharma A, et al. Diabetes mellitus and periodontal disease: the call for interprofessional education and interprofessional collaborative care - a systematic review of the literature. J Interprof Care 2020. 1-9. Advance online publication 10.1080/13561820.2020.1825354. 